\title{
Le principe de proportionnalité : retour sur quelques espoirs déçus
}

\section{Sébastien Van Drooghenbroeck et Xavier Delgrange}

\section{OpenEdition}

\section{Journals}

Édition électronique

URL : https://journals.openedition.org/rdr/290

DOI : $10.4000 /$ rdr.290

ISSN : 2534-7462

Éditeur

Presses universitaires de Strasbourg

\section{Édition imprimée}

Date de publication : 1 mai 2019

Pagination : 41-61

ISBN : 979-10-344-0045-4

ISSN : 2493-8637

Référence électronique

Sébastien Van Drooghenbroeck et Xavier Delgrange, «Le principe de proportionnalité : retour sur quelques espoirs déçus », Revue du droit des religions [En ligne], 7 | 2019, mis en ligne le 08 octobre 2019, consulté le 21 septembre 2021. URL : http://journals.openedition.org/rdr/290 ; DOI : https:// doi.org/10.4000/rdr.290

\section{(c) (†) (8)}

La revue du droit des religions est mise à disposition selon les termes de la Creative Commons Attribution - Pas d'Utilisation Commerciale 4.0 International - CC BY-NC 4.0. 


\section{LEPRINCIPEDE PROPORTIONNALITÉ: RETOURSURQUELQUESESPOIRSDÉÇUS}

\section{Sébastien VAN DROOGHENBROECK et Xavier DELGRANGE}

Université Saint-Louis - Bruxelles, Assesseur à la section de législation du Conseil d'État de Belgique;

Université Saint-Louis - Bruxelles et Université libre de Bruxelles, Premier auditeur chef de section au Conseil d'État de Belgique

\section{Résumé}

Le principe de proportionnalité occupe une place cardinale dans le raisonnement juridique toutes les fois qu'il s'agit d'apprécier la licéité d'une action ou d'une abstention au regard des normes protectrices des droits et libertés, en ce compris de la liberté de religion. Assez paradoxalement au regard de cette centralité, son contenu précis est longtemps demeuré assez mystérieux. Une reconstruction doctrinale macroscopique est aujourd'hui possible. Elle réduit les angles morts, mais ne les élimine pas complètement. La présente contribution s'efforce d'offrir un bilan de la matière, essentiellement au regard de la jurisprudence de la Cour européenne des droits de l'homme consacrée à l'article 9 de la Convention.

\section{Abstract}

The proportionality principle plays a key role in legal reasoning whenever the validity of an act or omission must be assessed in the light of norms protecting fundamental rights and freedoms, including the freedom of religion. Paradoxically, its exact content remained for a long time unclear, despite its central importance. A macroscopic doctrinal reconstruction is now possible. It reduces the blind spots, without, however, completely removing them. The purpose of this paper is to provide an overview of this question, essentially in relation to the case law of the European Court of Human Rights on article 9 of the Convention. 
L e principe de proportionnalité est a priori d'une simplicité confondante dans sa compréhension et dans son maniement. Il suffit, pour s'en convaincre, d'apercevoir le foisonnement d'images fortes et limpides qu'il convoque immédiatement dans l'esprit du juriste. Pour les uns, il convient de ne pas abattre des moineaux ${ }^{1}$, ou encore des mouches ${ }^{2}$, avec un canon. Dans la jurisprudence anglo-saxonne, on affirme qu'il ne faut pas casser des noix avec un marteau-pilon ${ }^{3}$. D'autres encore placent la proportionnalité en équation avec l'idée selon laquelle il ne faut pas arracher le bon grain avec l'ivraie ${ }^{4}$. On le voit: la proportionnalité semble entretenir une étroite intimité avec les maximes les mieux éprouvées de l'agir humain, et n'a nul besoin $\mathrm{du}$ jargon juridique pour se faire comprendre. Cette simplicité expliquerait que la proportionnalité ait pu si facilement conquérir les différents ordres juridiques et, en leur sein, les différentes branches du droit, et s'ériger en une sorte d'esperanto juridique, jalon d'un droit commun mondial.

Simple, la proportionnalité serait également sympathique. Elle offre aux acteurs du champ juridique la possibilité de réaliser au grand jour le vieux rêve d'équité qui, jusqu'alors, n'avait pu survivre que dans la clandestinité d'un droit dur et formel en se dissimulant sous les oripeaux trop ajustés du syllogisme judiciaire. Dans l'Abwägungsstaat de Walter Leisner ${ }^{5}$, ce sont les circonstances propres à chaque cause, et non les cas-types généraux et abstraits envisagés par le législateur, qui forment ultimement la matrice du droit valide. À la règle de fer rigide, la proportionnalité substituerait la règle de plomb, souple, infiniment respectueuse de l'irréductible singularité des situations et des êtres. À tout juge, elle offre la possibilité de dévoiler, sans rougir, le bon Président Magnaud qui sommeille sous sa robe; à tout justiciable, elle promet un droit sur mesure, bienveillant.

La séduction ainsi exercée par le principe de proportionnalité s'éprouverait, entre autres, sur le terrain délicat de la régulation des expressions convictionnelles et de l'aménagement juridique de la diversité religieuse. En particulier, l'exigence de «nécessité » et les impératifs de diligence procédurale, que la proportionnalité recèle en ses flancs, formeraient les véhicules

1. C. Debbasch, Préface in X. Philippe, Le contrôle de proportionnalité dans les jurisprudences constitutionnelle et administrative françaises, Paris, PUAM/Economica, 1990.

2. J. Velaers, Van Arbitragehof tot grondwettelijk Hof, Anvers, Makklu, 1990, p. 181.

3. R. v. Goldsmith (1983), 1 WLR 151, 155, per Lord Diplock.

4. O. MAYer, Deutsches Verwaltungsrecht, $3^{e}$ éd. 1925, t. I, p. 224, cité par M. Fromont, «Le principe de proportionnalité», AJDA 1995, p. 157.

5. W. LeISNER, Der Abwägungsstaat. Verhältnismassigkeit als Gerechtigkeit?, Berlin, Duncker \& Humblot, 1997. 
juridiquement corrects et «dicibles» d'une recherche d'accommodements raisonnables.

Les attentes ainsi formées à l'adresse de la proportionnalité nous semblent pouvoir être honorées, à tout le moins jusqu'à un certain point.

Certes, la présente contribution n'a pas l'ambition de faire une théorie générale de ce principe susceptible d'être validée dans les - très - nombreuses sphères juridiques, nationales ou internationales, de son apparition. Un double resserrement sera opéré. Tout d'abord, les lignes qui suivent seront essentiellement issues d'une observation du droit de la Convention européenne des droits de l'homme et de la jurisprudence de la cour éponyme, sans exclure, plus marginalement, l'un ou l'autre regard comparatiste jeté sur la production de l'autre juge européen. Ensuite, et au sein de la jurisprudence strasbourgeoise, l'accent sera principalement mis sur les précédents relatifs à la liberté de conscience et de religion et la mise en ouvre de l'exigence de proportionnalité qui conditionne la licéité des limitations qui affectent cette liberté.

$\mathrm{Au}$ sein du matériau ainsi circonscrit, une reconstruction doctrinale «macroscopique» permet d'apercevoir, derrière l'invocation générique de la proportionnalité, un système cohérent et assurément fécond d'exigences matérielles et formelles/procédurales (1). Tant s'en faut cependant que ce système, tout adéquat soit-il pour étancher la soif d'ordre du professeur, soit à tout coup mis en œuvre, sur le plan «microscopique» des espèces, par le juge: des éclipses et des angles morts subsistent, nonobstant les progrès méthodologiques réalisés au cours des dernières années, et ce, tant au niveau des exigences matérielles qu'au niveau des impératifs procéduraux coiffés par l'idée générique de proportionnalité (2). Comme l'écrivait F. Sudre en 2017, «le contrôle de proportionnalité exercé par la Cour européenne [des droits de l'homme] obéit à une démarche casuistique qui, là comme ailleurs, témoigne de la réticence de la Cour à se laisser enfermer dans des constructions théoriques préétablies ${ }^{6} »$. Une année plus tard, É. Dubout ${ }^{7}$ mettait en lumière - tout un paradoxe... - le «côté obscur» de la proportionnalité.

6. F. SUDRE, «Le contrôle de proportionnalité par la Cour européenne des droits de l'homme. De quoi est-il question?», JCP E, 13 mars 2017, p. 505.

7. É. Dubout, «Le côté obscur de la proportionnalité», in Les droits de l'homme à la croisée des droits. Mélanges en l'honneur de Frédéric Sudre, Paris, LexisNexis, 2018, p. 183-193. 


\section{LE "SYSTÈME » RÉVÉLÉ PAR L’ANALYSE «MACROSCOPIQUE »"}

«Il n'est pas de liberté sans ordre, ni plus d'ordre sans liberté», rappelle P. Abravanel ${ }^{9}$. La préservation de l'intérêt général et des intérêts d'autrui n'est pas l'«Autre» des droits et libertés, mais, dans une très large mesure, une condition de leur effectivité. Point de manichéisme étroit, mais une subtile dialectique faite de la recherche constante d'un équilibre entre les biens juridiques rivalisant. Le symptôme de cette recherche d'équilibre s'aperçoit dans la relativité des droits consacrés: à de très rares exceptions près, chacun d'entre eux s'expose à une ou plusieurs formes de limitations, qu'il s'agisse de restrictions ou d'exceptions - pour les temps normaux - ou de dérogations ou de déchéances - pour les circonstances extrêmes. L'outil de la recherche d'équilibre se présente, pour sa part, sous les traits de la proportionnalité, comme condition de validité omniprésente des limitations pareillement autorisées. Tel est le cas notamment pour la Convention européenne des droits de l'homme, telle qu'interprétée par la Cour ${ }^{10}$.

Comme souvent, la surconsommation d'un même terme par le discours juridique n'en facilite pas la définition, voire alimente le soupçon de sa relative vacuité. À force de vouloir tout dire, la proportionnalité ne signifierait-elle donc plus rien du tout? L'affirmer serait excessif. En effet, derrière cette condition de validité générique, peuvent être aperçues, avec plus ou moins de netteté - et au prix, parfois, d'une certaine patience - des exigences substantielles (1.1) et des exigences procédurales, formelles (1.2)

8. Les lignes qui suivent sont inspirées de S. Van Drooghenbroeck et C. Rizcallah, «Article $52 »$, in F. Picod et S. Van Drooghenbroeck (dir.), avec la collab. de C. Rizcallah, La Charte des droits fondamentaux de l'Union européenne. Commentaire article par article, Bruxelles, Larcier, 2017, p. 1083-1111. V. aussi X. Delgrange, "Une nouvelle source du droit: le dress code», in Y. CARTuYvels et al. (dir.), Le droit malgré tout. Hommage à François Ost, Publ. de l'Université Saint-Louis-Bruxelles, 2018, p. 659-706.

9. P. Abravanel, La protection de l'ordre public dans l'État régi par le droit, Bâle, Helbing et Lichtenhän, 1980, p. 145.

10. V. S. Van Drooghenbroeck, La proportionnalité dans le droit de la Convention européenne des droits de l'Homme. Prendre l'idée simple au sérieux, Bruxelles, Bruylant/FUSL, 2001, p. 71 et s. 
Le principe de proportionnalité : retour sur quelques espoirs déçus

\subsection{LES EXIGENCES SUBSTANTIELLES}

Issue du droit public allemand ${ }^{11}$ et éclairée par les travaux pionniers de Robert Alexy ${ }^{12}$, la recomposition théorique la plus pure du principe de proportionnalité aboutit à y apercevoir, tout d'abord, trois exigences d'ordre substantiel - i.e., portant sur le contenu même de la mesure querellée -, vérifiées en cascade. Les conclusions rendues respectivement par les avocats généraux Kokkott et Saugmandsgaardøe dans les affaires Achbita ${ }^{13}$ et Tele2 Sverige $A B^{14}$, sont, sur ce point, dignes des manuels les plus pédagogiques. Il faut d'abord que la mesure soit «appropriée» à la poursuite de l'objectif poursuivi, c'est-à-dire, qu'elle soit au moins « apte à contribuer ${ }^{15}$ » à sa réalisation. La mesure doit, ensuite, être «nécessaire » à la poursuite dudit objectif, ce qui ne peut être le cas «qu'en l'absence de toute autre mesure qui serait aussi appropriée tout en étant moins contraignante ${ }^{16} »$. Enfin, et à la supposer «appropriée» et «nécessaire» aux sens prédéfinis, la mesure devra encore être "proportionnée», au sens strict, à la poursuite de l'objectif poursuivi, ce qui implique que «les inconvénients causés ne sont pas démesurés par rapport aux buts visés ${ }^{17}{ }$.

Toujours selon les meilleurs manuels, chacune des trois exigences ainsi mise en lumière présente un contenu et une utilité propres dans le raisonnement, en sorte que nulle n'a vocation à s'éclipser derrière l'autre. Comme le souligne l'avocat général Saugmandsgaardøe,

«À la différence des exigences relatives au caractère approprié et nécessaire de la mesure en cause, lesquelles évaluent son efficacité au regard de l'objectif poursuivi, l'exigence de proportionnalité stricto sensu consiste à mettre en balance, d'une part, les avantages résultant de cette mesure au regard de l'objectif légitime poursuivi avec, d'autre

11. V. e.a., R. Deschling, Das Verhältnismassigkeitsgebot. Eine Bestandsaufnahme der Literatur zur Verhältnismässigkeit staatlichen Handels, München, Franz Vallen, 1989. On trouvera également, dans le droit constitutionnel suisse (lui-même fortement inspiré du droit allemand), une conception particulièrement «pure» de la proportionnalité. V. J. DuBEY, Droits fondamentaux, vol. 1, Notion, garantie, restriction et juridiction, Bâle, Helbing Lichtenhahn, 2018, p. 211 et s.

12. R. Alexy, Theorie der Grundrechte, Baden-Baden, Nomos, 1985.

13. Concl. de l'avocat général Kokott du 31 mai 2016 dans l'affaire CJUE, C-157/15, Achbita, pt. 97.

14. Concl. de l'avocat général Saugmandsgaardøe du 19 juillet 2016 dans l'affaire CJUE, C-203/15 et C-698/15, Tele2 Sverige AB, pt. 174.

15. Ibid., pt. 176 .

16. Ibid., pt. 185 .

17. Ibid., pt. 247. 
part, les inconvénients en découlant au regard des droits fondamentaux consacrés dans une société démocratique [...]. Cette exigence ouvre ainsi un débat sur les valeurs devant prévaloir dans une société démocratique et, en définitive, sur le type de société dans lequel nous souhaitons vivre $[\ldots]^{18}$ ».

Loin s'en faut toutefois que la pratique des juges ait présenté - par le passé $^{19}$ - et présente encore - à l'heure actuelle - la clarté des meilleurs manuels. Tel est le cas pour les juges de Luxembourg. Dans certains cas en effet, la Cour de Justice statue sur le respect du principe de proportionnalité sans réel raisonnement ${ }^{20}$. Dans d'autres cas, ledit principe est contracté autour des seules exigences de l'appropriation et de la nécessité ${ }^{21}$. Ce n'est que plus rarement que l'on voit poindre, en sus, l'exigence de la proportionnalité au sens strict ${ }^{22}$, dont la légitimité même fut d'ailleurs contestée avec véhémence, il y a de cela quelques années, par certains auteurs ${ }^{23}$. Entre les épures doctrinales visiblement inspirées du droit public allemand et le law in action du prétoire luxembourgeois, il y a donc une importante marge ${ }^{24}$.

La conclusion n'est pas différente s'agissant de la jurisprudence de la Cour européenne des droits de l'homme. Longtemps, celle-ci n'a même donné aucune réelle prise à un consensus doctrinal, ne fût-ce qu'élémentaire, sur le contenu du principe de proportionnalité. Au-delà de l'idée fort fruste d'un «juste équilibre» des intérêts en présence - formulée pour la première fois dans l'affaire linguistique belge ${ }^{25}$, rien de précis et d'incontestable ne pouvait être déduit sur ce thème ${ }^{26}$. Ce n'est qu'à la faveur d'une jurisprudence récente que les choses se sont quelque peu cristallisées, à tout le moins sur le plan des principes.

18. Ibid., pt. 248. V. aussi l'exemple de la note 81.

19. W. VAn Gerven, «The Effect of Proportionality on the Actions of Member States of the European Community: National Viewpoints from Continental Europe», in E. Ellis (dir.), The principle of proportionality in the laws of Europe, Oxford, Hart, 1999, p. 37.

20. CJUE, $1^{\text {er }}$ mars 2011, aff. C-236/09, Association Belge des Consommateurs Test-Achats.

21. CJUE, 9 nov. 2010, aff. C-92/09 et C-93/09, Volker und Schecke, pt. $50 ; 17$ oct. 2013, aff. C-291/12, Schwarz, pt. 34 et 40; 8 avril 2014, aff. C-293/12 et C-594/12, Digital Rights Ireland Ltd, pt. 46.

22. W. VAN GERVEn, op. cit., p. 37.

23. S. Van Drooghenbroeck, La proportionnalité..., op. cit., p. 224, note 218.

24. V. M. Arden, «Proportionality: the Way Ahead?», Human Rights and European Law. Building New Legal Orders, Oxford, OUP, 2015, p. 56 et s.

25. CEDH, 23 juill. 1968, Affaire relative à certains aspects du régime linguistique de l'enseignement en Belgique, \& 5.

26. S. Van Drooghenbroeck, La proportionnalité..., op. cit., p. 345-348. 
L'exigence d'appropriation s'aperçoit aujourd'hui de manière relativement nette dans la jurisprudence dédiée à la prohibition de diffusion d'informations «publiques ${ }^{27}$, formée sous visa de l'article 10 de la Convention, ainsi que dans le contentieux de l'égalité. Selon l'arrêt Vallianatos et autres c. Grèce,

«lorsque la marge d'appréciation laissée aux États est étroite, dans le cas par exemple d'une différence de traitement fondée sur le sexe ou l'orientation sexuelle, non seulement le principe de proportionnalité exige que la mesure retenue soit normalement de nature à permettre la réalisation du but recherché, mais il oblige aussi à démontrer qu'il était nécessaire, pour atteindre ce but, d'exclure certaines personnes en l'espèce les individus vivant une relation homosexuelle - du champ d'application de la mesure dont il s'agit [...]. En vertu de la jurisprudence précitée, la charge de cette preuve incombe au gouvernement défendeur ${ }^{28}$ \%.

Tout en ne contestant pas que l'exigence de proportionnalité emporte effectivement une condition d'appropriation ${ }^{29}$, l'arrêt Garib c. Pays-Bas précise que la satisfaction à cette condition doit être évaluée à la lumière des données disponibles au moment où est intervenue la décision litigieuse, et non, avec le bénéfice du recul, à la lumière des données révélées ultérieurement ${ }^{30}$. Cette position, tout en n'étant pas totalement inédite dans la jurisprudence strasbourgeoise, n'y est cependant pas toujours assumée avec toute la cohérence voulue ${ }^{31}$.

Ainsi que le laisse apercevoir l'extrait précité de l'arrêt Vallianatos, l'exigence de proportionnalité emporte, de manière supplémentaire, la condition de nécessité, au sens où nous l'avons définie ci-dessus. C'est sur cet aspect des choses que - sans aucun doute - l'accouchement aura été le plus difficile. Longtemps en effet, la jurisprudence strasbourgeoise a nourri la problématique d'indices parfaitement contradictoires ${ }^{32}$; nous verrons au demeurant que ce n'est pas totalement fini. En 2009 encore, J. Christoffersen ${ }^{33}$ pouvait énoncer, en préambule d'une analyse jurisprudentielle particulièrement fouillée, que la Cour européenne des droits de l'homme avait clairement

27. Ibid., p. 179-180.

28. CEDH, 7 nov. 2013, Vallianatos et a. c. Grèce, § 85.

29. V. aussi CEDH, 28 août 2012, Costa et Pavan c. Italie, \& 84.

30. CEDH, Gde ch., 6 nov. 2017, Garib c. Pays-Bas, § 147.

31. V. S. Van Drooghenbroeck, La proportionnalité..., op.cit., p. 185-187.

32. Ibid., p. 190-203.

33. J. Christoffersen, Fair Balance: Proportionality, Subsidiarity and Primarity in the European Convention on Human Rights, Leiden, Martinus Nijhoff, 2009. 
rejeté l'idée d'inclure, dans l'exigence de proportionnalité, l'obligation pour les États de choisir l'alternative la moins restrictive pour parvenir au but poursuivi ${ }^{34}$.

C'est précisément en 2009 que, pour la toute première fois à notre connaissance, la Cour européenne des droits de l'homme franchit le Rubicon en affirmant de manière générale et abstraite que "pour qu'une mesure puisse être considérée comme proportionnée et nécessaire dans une société démocratique, l'existence d'une mesure portant moins gravement atteinte au droit fondamental en cause et permettant d'arriver au même but doit être exclue ${ }^{35}$ ».

Issu de l'arrêt Glor c. Suisse, ce dictum fut par la suite réitéré à de nombreuses reprises ${ }^{36}$. On en trouvera une mise en œuvre significative dans l'arrêt Osmanoglu et Kocabas c. Suisse du 10 janvier 2017 37 . En l'espèce, la Cour était amenée à examiner si le refus des autorités compétentes d'exempter les filles des requérants, de confession musulmane, des cours de natation mixtes, était «nécessaire dans une société démocratique»et, plus particulièrement, proportionné aux buts poursuivis par ces mêmes autorités. Sur ce terrain ${ }^{38}$, les requérants soutenaient que l'obligation pour leurs filles de suivre des cours de natation mixtes n'était pas appropriée et que la participation à ces cours n'était pas requise pour atteindre les objectifs invoqués, à savoir leur formation et leur intégration, ainsi qu'un fonctionnement scolaire ordonné et efficace. Ils estimaient que l'objectif de formation visé par le cours de natation pouvait être atteint avec des moyens plus modérés, qui subordonneraient par exemple l'octroi d'une dispense à l'obligation des parents de faire suivre à leurs enfants des cours de natation à titre privé. Par ailleurs, ils estiment que le port du burkini - qui était autorisé - ne contribuerait pas à la solution du problème en cause au motif qu'il stigmatiserait leurs filles. Le plaidoyer ainsi

34. Ibid., p. 114.

35. CEDH, 30 avril 2009, Glor c. Suisse, § 94.

36. V. e.a., CEDH, 22 déc. 2009, Sejdić and Finci c. Bosnie-Herzégovine, § $48 ; 11$ oct. 2011, Association Rhino et autres c. Suisse, § 65; 21 juin 2012, Schweizerische Radio- und Fernsehgesellschaft SRG c. Suisse, § 61; 13 juill. 2012, Mouvement raëlien suisse c. Suisse, $\S 75$; 12 sept. 2012, Nada c. Suisse, § 183; 16 janv. 2014, Abdulayeva c. Russie, § 34; 12 juin 2014, Biblical Centre of the Chuvash Republic c. Russie, § 58; 29 mai 2018, Gulbahar Ozer et Yusuf Ozer c. Turquie, § 34; 6 juin 2013, Sabanchiyeva et autres c. Russie, § 133; 4 déc. 2015, Roman Zakahrov c. Russie, § 260 et réf. citées; 7 févr. 2017, Lashmankin et autres c. Russie, § 373; 18 avr. 2013, Saint-Paul Luxembourg S.A. c. Luxembourg, § 44. V. encore E. Brems et L. LAVRYSEn, «'Don't Use a Sledgehammer to Crack a Nut': Less Restrictive Means in the Case Law of the European Court of Human Rights», Human Rights Law Review, 15 (1), 2015, p. 1-30.

37. CEDH, 10 janv. 2017, Osmanoglu et Kocabas c. Suisse.

38. Ibid., \& 66 . 
livré, relevant clairement du registre de la «non-nécessité» de la mesure, fut repoussé par la Cour. À son estime en effet, l'intérêt de l'enseignement obligatoire de la natation «ne se limite pas pour les enfants à apprendre à nager et à exercer une activité physique, mais il réside surtout dans le fait de pratiquer cette activité en commun avec tous les autres élèves, en dehors de toute exception tirée de l'origine des enfants ou des convictions religieuses ou philosophiques de leurs parents ${ }^{39}$ ». Au regard de l'objectif ainsi défini, l'alternative moins restrictive suggérée par les requérants - accorder une dispense aux enfants qui suivent des cours de natation privés - n'en était en réalité pas une: le but de socialisation et - oserait-on dire avec un mauvais jeu de mots -, de «brassage convictionnel», n'aurait pas été atteint. Tout au contraire, «exempter des enfants dont les parents ont des moyens financiers suffisants pour leur assurer un enseignement privé créerait par rapport aux enfants dont les parents ne disposent pas de tels moyens une inégalité non admissible dans l'enseignement obligatoire ${ }^{40} »$. Plus avant, la Cour affirme que la Suisse s'est efforcée de réduire les atteintes aux droits des requérants au strict minimum:

«dans la présente affaire, les autorités ont offert des aménagements significatifs aux requérants, dont les filles avaient notamment la possibilité de couvrir leurs corps pendant les cours de natation en revêtant un burkini. Or les requérants ont soutenu que le port du burkini avait un effet stigmatisant sur leurs filles. Sur ce point, la Cour partage l'avis du Gouvernement selon lequel les requérants n'ont apporté aucune preuve à l'appui de leur affirmation. Elle note que, par ailleurs, les filles des requérants pouvaient se dévêtir et se doucher hors de la présence des garçons. Elle admet que ces mesures d'accompagnement étaient à même de réduire l'impact litigieux de la participation des enfants aux cours de natation mixtes sur les convictions religieuses de leurs parents ${ }^{41}$.»

Des «aménagements significatifs...». Le vocabulaire utilisé ne trompe pas. Ainsi que le laissait entendre l'arrêt Jakobski c. Pologne ${ }^{42}$, l'exigence de nécessité - lorsqu'elle est vérifiée - conduit à s'interroger en substance, sans cependant utiliser les mots qui fâchent, sur les «accommodements

39. Ibid., \& 98 .

40. Ibid., \& 100 .

41. Ibid., § 101 .

42. CEDH, 7 déc. 2010, Jakobski c. Pologne. V. aussi CEDH, 17 déc. 2013, Vartic c. Roumanie $\left(n^{\circ} 2\right)$, ainsi que S. OuAld CHAiB, «Gatis Kovalkovs v. Latvia: The Strasbourg Court keeps the door to reasonable accommodation open $»$ : https://strasbourgobservers.com [consulté le 18 déc. 2018]. 
raisonnables» disponibles ${ }^{43}$. Pour le dire avec les mots des juges Tulkens, Keller et Popovic ${ }^{44}$, «la recherche d'un aménagement raisonnable de la situation litigieuse peut, dans certaines circonstances, constituer un moyen moins restrictif d'atteindre l'objet poursuivi».

L'exigence générique de proportionnalité strasbourgeoise coiffe enfin, sur le plan substantiel, la condition de proportionnalité au sens strict. Il s'agit de «mettre en balance», cette fois-ci dans le registre axiologique, les intérêts conflictuels pour déterminer celui qui, en l'occurrence, pèse le plus lourd. Cette ultime étape du raisonnement proportionnaliste est celle qui, dans le raisonnement de la Cour, est d'emblée apparue la plus évidente. Au vrai, cette évidence est relativement paradoxale, car cette "pesée des intérêts» ne va en réalité nullement de soi. Elle requiert en effet, pour la détermination des intérêts à peser et du poids à leur conférer, un «cadrage» strict du litige, obtenu par le positionnement sur différents axes ${ }^{45}$. Lorsqu'est contestée une mesure d'application d'une législation plus générale, faut-il opérer la pesée des intérêts au niveau de la législation elle-même - un categorical balancing - et confronter alors les préjudices et bénéfices généraux attachés à cette législation, ou faut-il opérer au contraire une pesée des intérêts en présence dans la situation d'application proprement dite - un ad hoc balancing - et ne confronter que des préjudices et des bénéfices concrets, observables dans la situation d'espèce? Autre question cruciale: les intérêts doivent-ils être pondérés ex tunc, c'est-à-dire, au jour où la mesure querellée a été adoptée, ou doivent-ils être pondérés ex nunc, c'est-à-dire, au jour où le juge tranche le litige? La Cour européenne des droits de l'homme n'a jamais totalement théorisé ces questions méthodologiques, et ne leur apporte donc pas de

43. V. dans ce numéro X. Delgrange et H. Lerouxel, «L'accommodement belge éclot entre égalité formelle, légalité et neutralité». V. aussi des mêmes auteurs, "L'accommodement raisonnable, bouc émissaire d'une laïcité inhibitrice», in E. BRIBOSIA et I. RORIVE (dir.), L'accommodement raisonnable de la religion en Belgique et au Canada. Comparaison des contextes juridiques, sociaux et politiques, Bruxelles, Peter Lang, 2015, p. 203-286, spéc. p. 254-263; E. Bribosia, J. Ringelheim et I. RoRive, «Aménager la diversité: le droit de l'égalité face à la pluralité religieuse», RTDH 2009, p. 319 et s. V. encore E. BRIBOSIA et I. RORIVE, «Les droits fondamentaux, gardiens et garde-fous de la diversité religieuse en Europe», in L'accommodement raisonnable de la religion en Belgique et au Canada, op. cit., p. 171-202, spéc. p. 202.

44. Opinion dissidente jointe à CEDH, 3 avr. 2012, Francesco Sessa c. Italie.

45. V. S. Van Drooghenbroeck, La proportionnalité..., op. cit., p. 247 et s.; G. Willems et A. Hoc, «Le cadrage temporel des litiges devant la Cour européenne des droits de l'homme: réflexion au départ de l'arrêt S.H. et autres c. Autriche du 3 novembre 2011 », in Le temps et le droit. Hommage au Professeur Closset-Marchal, Bruxelles, Bruylant, 2013, p. 283-308. 
réponses tout à fait cohérentes ${ }^{46}$. Qu'il suffise, pour s'en convaincre, de relire les opinions dissidentes jointes à l'arrêt de Grande Chambre intervenu dans l'affaire Garib c. Pays-Bas ${ }^{47}$. Ainsi que le rappelle É. Dubout ${ }^{48}$, une autre illusion consiste à faire «comme si » les intérêts mis en balance pro et contra présentaient une «commensurabilité» qui permettrait de déterminer que l'un est objectivement plus lourd que l'autre. Cette commensurabilité suppose l'existence d'une «métrique» commune, d'un tertium comparationis, laquelle est évidemment pure chimère, en éthique comme en droit ${ }^{49}$. Feu A. Scalia, juge à la Cour suprême américaine, résumait excellemment la situation: procéder à une balance des intérêts, c'est comme juger que «telle ligne est plus longue que telle pierre est lourde ${ }^{50} »$. L'incommensurabilité des intérêts en présence n'évite donc pas à la balance des intérêts d'être le lieu d'une irréductible projection des hiérarchies de valeurs personnelles du juge.

\subsection{LES EXIGENCES PROCÉDURALES/FORMELLES}

Appropriation, nécessité, proportionnalité au sens strict: voilà qui forme le volet substantiel. Il apparaît cependant que, sous le label générique de la «proportionnalité», la Cour européenne des droits de l'homme intègre également des exigences de type procédural, formel. Pareille opération est elle-même un sous-produit du phénomène dit de "procéduralisation» des droits substantiels garantis par la Convention européenne des droits de l'homme, phénomène auquel une attention doctrinale massive a été consacrée au cours des dernières années ${ }^{51}$.

46. V. S. Van Drooghenbroeck, La proportionnalité..., op.cit., p. 247 et s.; V. aussi S. Van Drooghenbroeck, F. Krenc et O. Van der Noot, «Questions of Method: the Use of 'external sources' in National Union of Rail, Maritime and Transport Workers $v$. the United Kingdom», in E. BREMS and E. DeSMET (eds), Integrated Human Rights in Practice. Rewriting Human Rights Decisions, Cheltenham, Edward Elgar, 2017, p. 48.

47. V. spéc. l'opinion dissidente du juge Kuris jointe à CEDH, 6 nov. 2017, Garib c. Pays-Bas.

48. É. Dubout, «Le côté obscur...», op.cit., p. 189 et s.

49. V. R. Chang, Incommesurabilty, Incomparability and Practical Reasoning, Cambridge, Harvard University Press, 1997. - V. également Law and Incommensurability, Symposium publié dans University of Pennsylvania Law Review, vol. 146, 1998.

50. Bendix Autolite Corp. v. Midwesco Enterprises, 488 US 888, 897 (1988), Scalia J. concurring [traduction libre].

51. V. e.a., parmi les travaux les plus récents, N. Le BonNiEC, La procéduralisation des droits substantiels par la Cour européenne des droits de l'homme. Réflexion sur le contrôle juridictionnel des droits garantis par la Convention européenne des droits de l'homme, Bruxelles, Larcier, 2017; E. BREMS et J. GERARds (dir.), Procedural Review in European Fundamental Rights Cases, Cambridge University Press, 2017; O. M. ARNARdótTiR, «The "procedural turn' under the European Convention on Human Rights and presumptions of Convention 
Cette procéduralisation est protéiforme, et, de ce fait même, particulièrement périlleuse à définir. En grosse approximation, on dira qu'elle consiste à tirer d'entre les lignes des dispositions conventionnelles garantissant des droits substantiels une série d'impératifs procéduraux dont le respect conditionne la licéité des restrictions aux droits qu'elles consacrent ou des abstentions qui, sous leur visa, sont reprochées à l'État. Dans la perspective du mouvement procédural, c'est moins le fond des décisions qui importe, que la manière dont on a décidé, ou permis que la décision soit contestée ex post.

Une des multiples manifestations de la «procéduralisation» de l'exigence de proportionnalité consiste à requérir que toute décision individuelle portant restriction aux droits et libertés s'accompagne de garanties contre l'arbitraire, en amont de la décision - audi alteram partem, devoir de motivation... - et en aval de la décision: un contrôle juridictionnel effectif doit pouvoir être enclenché. L'arrêt Osmanoglu et Kocabas c. Suisse du 10 janvier 2017, ci-avant évoqué, est significatif à cet égard. Sous visa de l'exigence de «nécessité dans une société démocratique» de l'ingérence querellée, la Cour évoque en effet « la mise en place d'une procédure effective et accessible en vue de protéger les droits garantis par [l'article 9 de la Convention européenne des droits de l'homme], et notamment la création d'un cadre réglementaire instaurant un mécanisme judiciaire et exécutoire destiné à protéger les droits des individus et la mise en œuvre, le cas échéant, de mesures spécifiques appropriées ${ }^{52}$ ». En l'occurrence, la Cour estima qu'il était satisfait aux impératifs procéduraux pareillement imposés:

«les autorités ont publié une directive sur le traitement à réserver aux questions religieuses à l'école, dans laquelle les requérants ont pu trouver les informations pertinentes [...]. Puis l'autorité compétente a averti les requérants qu'ils encouraient une amende si leurs enfants n'étaient pas présents aux cours de natation obligatoires [...]. À la suite d'un entretien avec la direction de l'école et de deux lettres adressées par celle-ci aux requérants, l'autorité compétente a infligé aux requérants les amendes qui étaient prévues par le droit interne pertinent $[\ldots]$ et

compliance», International Journal of Constitutional Law, 2017, p. 9-35; P. Popelier et C. van DE Heyning, «Subsidiarity Post-Brighton: Procedural Rationality as Answer? », Leiden Journal of International Law 2017, p. 5-23; L.M. HuijBers et J.H. GERARDS, « Procedurele toetsing », in R. ORTLEP et al., De rechter onder vuur, Oisterwijk, Wolf Legal Publ., 2016, p. 191-122; L. M. HUiJBERS, "The European Court of Human Rights' procedural approach in the age of subsidiarity ", Cambridge International Law Journal, (6) 2017, p. 177-2011; M. SAUL, «The European Court of Human Rights' Margin of Appreciation and the Processes of National Parliaments ", Human Rights Law Review 2015, p. 745-774.

52. CEDH, 10 janv. 2017, Osmanoglu et Kocabas c. Suisse, § 86. 
que les intéressés ont pu contester devant la cour d'appel du canton de Bâle-Ville, puis devant le Tribunal fédéral. À l'issue de procédures équitables et contradictoires, ces deux juridictions, dans le cadre de décisions dûment motivées, sont arrivées à la conclusion que l'intérêt public consistant à suivre de manière intégrale le programme scolaire obligatoire devait prévaloir sur l'intérêt privé des requérants d'obtenir pour leurs filles une dispense des cours de natation mixtes ${ }^{53}$ ».

Une autre manifestation de ce mouvement de procéduralisation consiste en l'examen, par la Cour européenne des droits de l'homme, de la qualité du processus parlementaire qui a conduit à l'adoption de telle ou telle législation qui, en son principe même ou dans son application concrète, se trouve querellée devant elle ${ }^{54}$.

Un exemple remarquable de cette déclinaison «parlementaire » du mouvement procédural est fourni par l'arrêt Hirst $\left(n^{\circ} 2\right) c$. Royaume-Uni, à propos de la suspension automatique du droit de vote des personnes détenues suite à une condamnation pénale. En soutien de la condamnation finalement intervenue sous le visa de l'article $3 \mathrm{du}$ premier protocole additionnel, la Cour énonça en effet que

«rien ne montre que le Parlement ait jamais cherché à peser les divers intérêts en présence ou à apprécier la proportionnalité d'une interdiction totale de voter visant les détenus condamnés. [...], on ne saurait dire que les députés ont tenu un débat de fond sur le point de savoir s'il se justifiait toujours, à la lumière de la politique pénale moderne et des normes en vigueur en matière de droits de l'homme, d'appliquer une telle restriction générale au droit de vote des détenus ${ }^{55}$ ».

Un autre exemple significatif ${ }^{56}$ est fourni par l'arrêt Parrillo c. Italie rendu en Grande Chambre le 27 août $2015^{57}$. La question qui se posait en l'espèce

53. Ibid., \& 104 .

54. V. par ex. CEDH, 20 juin 2017, Bayev et autres c. Russie, § 63. V. aussi CEDH, 23 mars 2017, A.-M V. c. Finlande, § 82; 21 févr. 2017, Orlovskaya Iskra c. Russie, § 126.

55. CEDH, Gde ch., 6 oct. 2005, Hirst c. Royaume-Uni ( $\left.n^{\circ} 2\right)$, § 79.

56. V. encore, e.a., CEDH, 3 nov. 2011, S.H. et a. c. Autriche, § 117-118; 27 juin 2017, Satakunnan Markikinaporssi Oy et Satamedia Oy c. Finlande, § 192-193; 21 févr. 1986, James et autres c. Royaume-Uni, \& 48; 10 avr. 2007, Evans c. Royaume-Uni, § 86; 22 avr. 2013, Animal Defenders International c. Royaume-Uni, \& 114-116; 7 mai 2013, Shindler c. Royaume-Uni, \& 117; 4 déc. 2007, Dickson c. Royaume-Uni, \& 78-84; 20 mai 2010, Alajos Kiss c. Royaume-Uni, § 41; 6 oct. 2005, Maurice c. France, § 121.

57. CEDH, Gde ch., 27 août 2015, Parrillo c. Italie; CEDH, 8 avr. 2014, National Union of Rail, Maritime and Transport Workers (RMT) c. Royaume-Uni; 4 juill. 2013, Anchugov et Gladkov c. Russie, § 108-109; 11 déc. 2014, Dubska et Krejzova c. République tchèque. 
était celle de la compatibilité avec l'article 8 de la Convention (droit au respect de la vie privée) de l'interdiction légale faite à la requérante de faire don, à des fins de recherche scientifique, de ses embryons issus d'une fécondation in vitro. La Cour répondit en l'occurrence de manière affirmative. En soutien de cette conclusion, elle fit valoir que

«lors du processus d'élaboration de la loi litigieuse, le législateur avait déjà tenu compte des différents intérêts ici en cause, notamment celui de l'État à protéger l'embryon et celui des personnes concernées à exercer leur droit à l'autodétermination individuelle sous la forme d'un don de leurs embryons à la recherche ${ }^{58}$ ».

L'argument était lui-même étayé, entre autres, par le constat que le débat parlementaire dont était issue l'interdiction litigieuse, avait été «enrichi par les contributions de médecins, spécialistes et associations engagées dans le domaine de la procréation médicalement assistée $[\ldots]^{59}$ ».

Encore que de manière plus implicite, l'arrêt S.A.S. c. France ${ }^{60}$ relatif à la dissimulation du visage dans l'espace public peut être épinglé, selon certains juges à la Cour, comme l'un de ceux où la qualité de la discussion préalable à la décision proprement dite aura contribué au verdict de conventionnalité de cette décision. Selon la juge Nussberger en effet,

«[The legislative] procedure, which was extensively described in the first part of the judgement $[\ldots]$ did not only matter, but was a dominant aspect of the case. In this sense, it may be argued that there is not only an explicit, but also an implicit procedural review. Whenever the Court accepts the 'choice of society' based on a democratic decision-making process, it can be assumed that in the Court's view, the procedure which led to this decision fulfilled all the requirements ${ }^{61} »$.

Ainsi donc, la proportionnalité d'une mesure est favorisée - sans pour autant être assurée ${ }^{62}$ - par un débat parlementaire, administratif ou judiciaire

58. CEDH, Gde ch., 27 août 2015, Parrillo c. Italie, § 188.

59. Ibid., § 185.

60. CEDH, Gde ch., $1^{\text {er }}$ juill. 2014, S.A.S c. France.

61. A. Nussberger, «Procedural Review by the ECHR: View from the Court», in E. Brems et J. Gerards, Procedural Review in European Fundamental Rights Cases, Cambridge University Press, 2017, p. 164. La juge allemande fait ici allusion au $\S 154$ de l'arrêt: «Or, dans un tel cas de figure, la Cour se doit de faire preuve de réserve dans l'exercice de son contrôle de conventionnalité dès lors qu'il la conduit à évaluer un arbitrage effectué selon des modalités démocratiques au sein de la société en cause».

62. Si approfondi soit-il, un débat parlementaire ne débouchera pas nécessairement, en logique comme en droit, sur une solution définitivement acceptable au regard de la Convention 
où l'ensemble des intérêts concernés auront pu se faire valoir, dans une logique ouverte de concessions réciproques: ici encore touche-t-on du doigt, sans en prononcer le mot, la logique des "accommodements raisonnables». Le lien ainsi tracé entre les accommodements raisonnables, l'exigence de nécessité et la procéduralisation du principe de proportionnalité s'aperçoit, de manière particulièrement nette, dans les avis $\mathrm{n}^{\text {os }} 59.484 / 3$ et 59.485/3 donnés le 29 juin 2016 par le Conseil d'État de Belgique à propos de propositions de décret visant à interdire, de manière pure et simple, l'abattage d'animaux sans étourdissement préalable ${ }^{63}$. À l'estime de la Section de législation, pareille interdiction absolue méconnaît notamment l'article 9 de la Convention européenne des droits de l'homme, faute de réaliser le juste équilibre des intérêts en présence. Les avis ajoutent cependant, dans une visée "thérapeutique», que des mesures alternatives seraient concevables qui, tout en poursuivant l'objectif de réalisation du bien-être animal, représenteraient une limitation admissible à la liberté de religion du fait qu'elles n'iraient pas jusqu'à impliquer l'interdiction totale d'abattage sans étourdissement. Et le Conseil d'État d'ajouter que pareilles mesures

«gagnent à être élaborées dans le dialogue, lequel suppose lui-même que chacune des deux parties se montre ouverte aux alternatives. Dans cette optique, l'audition qui a été organisée sur ce point par le Parlement flamand est une première étape, mais des démarches supplémentaires semblent être envisageables. Il appartiendra ultimement au législateur décrétal de déterminer un équilibre entre, d'une part, le respect dû à la

européenne des droits de l'homme (V. A. Nussberger, op.cit., p. 167). Ce débat peut en effet avoir été mené en des temps relativement anciens, et n'avoir jamais fait l'objet d'une actualisation depuis lors. Or, les exigences de la Convention ont fort bien pu évoluer dans l'intervalle. Par ailleurs, une majorité parlementaire - si imposante soit-elle - n'est pas nécessairement respectueuse des droits et libertés, et, singulièrement, des droits et libertés des minorités. C'est la raison pour laquelle la Cour ne peut jamais abdiquer toute forme de contrôle substantiel au profit d'une approche strictement procédurale (V. F. TUlKens, "Conclusions générales", in F. Sudre (éd.), Le principe de la subsidiarité au sens du droit de la Convention européenne des droits de l'homme, Limal, Anthemis 2014, p. 406). Comme le soulignent les juges Spano et Karakas (opinion séparée jointe à l'arrêt Belcacemi et Oussar c. Belgique du 11 juillet 2017), «l'histoire a amplement démontré que les sociétés démocratiques portent en elles le risque que des sentiments majoritaristes, qui se traduisent par la suite en textes législatifs, germent sur le terreau d'idées et de valeurs qui menacent les droits fondamentaux. Les catégories isolées et vulnérables n'ont alors plus que le recours de s'adresser aux tribunaux. Et ces tribunaux, qu'ils soient nationaux ou internationaux, à l'instar de la Cour, ont le devoir de rechercher et de détecter, dans la mesure du possible, si l'imposition de mesures qui ont pourtant été largement entérinées par la sphère législative est motivée par une hostilité ou une intolérance à l'égard d'une idée, d'une opinion, ou d'une confession religieuse en particulier».

63. Doc. VI. Parlement, 2014-2015, n 111/2. 
liberté de religion et, d'autre part, l'objectif de réduction de la souffrance animale, et ce, compte dûment tenu de la protection conventionnelle et constitutionnelle des droits fondamentaux ${ }^{64} »$.

\section{LES HÉSITATIONS, CONTRADICTIONS ET ANGLES MORTS RÉVÉLÉS PAR L'ANALYSE «MICROSCOPIQUE»}

Toute satisfaisante qu'elle soit d'un point de vue intellectuel et en termes de recherche de justice, la systématique décrite dans les lignes qui précèdent ne transparaît pas de chacun des décisions et arrêts du juge européen des droits de l'homme. Loin s'en faut. Tantôt l'exigence de nécessité est oubliée, voire contredite (2.1). Dans d'autres hypothèses, ce sont les prolongements procéduraux de l'exigence de proportionnalité qui passent à la trappe (2.2).

\subsection{LA CONDITION DE NÉCESSITÉ : ÉCLIPSES ET CONTRADICTIONS}

Il est des précédents notables où la Cour européenne des droits de l'homme a omis d'examiner l'existence d'une alternative moins restrictive à la mesure querellée devant elle. Tel fut le cas, entre autres, dans l'arrêt Francesco Sessa c. Italie ${ }^{65}$. L'affaire concernait la compatibilité avec l'article 9 de la Convention du refus de l'autorité judiciaire de reporter, à la demande d'un avocat de confession juive, une audience fixée le jour d'une fête juive. La Cour a notamment estimé que, même à supposer l'existence d'une ingérence dans le droit du requérant protégé par l'article 9, cette ingérence, prévue par la loi, se justifiait par la protection des droits et libertés d'autrui, et en particulier le droit des justiciables de bénéficier d'un bon fonctionnement de l'administration de la justice et le respect du principe du délai raisonnable de la procédure. Le raisonnement de la Cour est, en l'occurrence, particulièrement expéditif. Le contrôle de nécessité en est totalement absent. À l'estime de trois des sept juges ${ }^{66}$ ayant statué en l'espèce, il y aurait pourtant eu toute sa place:

«[...] nous pensons que les conditions étaient réunies pour tenter
d'arriver à un aménagement et un aménagement raisonnable - c'est-à-dire
qui n'entraîne pas pour les autorités judiciaires une charge dispropor-
tionnée - de la situation. Avec quelques concessions, celui-ci aurait
permis d'éviter une ingérence dans la liberté religieuse du requérant,

64. Ibid., p. 16 [traduction libre]. V. dans ce numéro X. Delgrange et H. LerouXel, «L'accommodement belge éclot entre égalité formelle, légalité et neutralité», p. 109.

65. CEDH, 3 avril 2012, Francesco Sessa c. Italie.

66. Opinion dissidente commune aux juges Tulkens, Popovic et Keller. 
sans pour autant compromettre la réalisation du but légitime que constitue de toute évidence la bonne administration de la justice.

Tout d'abord, affirment les juges dissidents, le requérant a immédiatement, dès le moment de la fixation de la date de l'audience, soulevé la difficulté qui était la sienne et demandé le report de celle-ci. Il a donc prévenu les autorités judiciaires quatre mois à l'avance, ce qui leur permettait raisonnablement d'organiser le calendrier des audiences afin de garantir le respect des différents droits en jeu.

[...]

Ensuite, poursuivent les juges Tulkens, Popov et Keller, il n'est pas démontré en l'espèce que la demande du requérant, si elle avait été acceptée, aurait provoqué une telle perturbation dans le fonctionnement du service public de la justice. [...] Certes, le report demandé de l'audience pouvait entraîner certains inconvénients administratifs, comme par exemple la nécessité de renouveler la notification de la date d'audience aux parties impliquées. Mais ceux-ci nous paraissent minimes et constituent peut-être le modique prix à payer pour le respect de la liberté de religion dans une société multiculturelle.»

À l'estime d'une partie de la doctrine ${ }^{67}$, ce genre d'éclipse du test de nécessité peut s'expliquer par l'ampleur de la marge d'appréciation implicitement concédée dans une affaire comme celle-là: lorsque cette marge est large, le respect des conditions d'adéquation et de nécessité est irréfragablement présumé, et ne fait donc logiquement pas l'objet d'un quelconque contrôle de la part de l'instance strasbourgeoise. Il y aurait donc lieu de distinguer une «règle de proportionnalité » - qui inclut effectivement l'exigence de nécessité - et un "contrôle de proportionnalité»-qui ne vérifie pas nécessairement le respect de cette exigence, en fonction de l'amplitude de la marge d'appréciation concédée.

Pareille reconstruction, que valide l'extrait précité de l'arrêt Vallianatos, n'est toutefois que d'un secours pratique limité - vu l'incertitude persistante qui subsiste quant aux facteurs qui déterminent l'amplitude de la marge nationale d'appréciation - et, plus grave encore, se trouve elle-même tenue en échec par la subsistance de positions affirmant que l'absence de "nécessité » d'une mesure, fût-elle même vérifiée, ne conduit pas systématiquement à la méconnaissance de l'exigence de proportionnalité ${ }^{68}$.

67. V. S. Van Drooghenbroeck, La proportionnalité..., op. cit., p. 171-174 et p. 510. V. aussi F. SUDRE, «Le contrôle de proportionnalité...», op.cit., p. 509-512.

68. Sur ceci, V. L. LAVRYSEN, "On sledgehammers and nutcrackers: recent developments in the Court's less restrictive means doctrine»: https://strasbourgobservers.com [consulté le 18 déc. 2018]. 
Pareille position fut récemment formulée sous la plume du juge Paul Lemmens, s'exprimant en marge de l'arrêt Gülbahar Özer and Ysuf Özer c. Turquie du 29 mai $2018^{69}$. Contemplant la jurisprudence Glor ci-devant rappelée, le juge belge affirme en effet que:

« this case-law is difficult to reconcile with the long-standing principle that the adjective 'necessary', within the meaning of Article 8 \& 2 (and similar Articles), is not synonymous with 'indispensable' (compare with the expressions 'absolutely necessary' (Article 2 \& 2), 'strictly necessary' (Article $6 \S 1$ ), and 'to the extent strictly required by the exigencies of the situation' (Article $15 \& 1$ )) (Handyside v. the United Kingdom, 7 December 1976, \& 48, Series A no. 24) [...]).

[...] the central question as regards the necessity of an interference is not whether a less restrictive measure should have been adopted or, indeed, whether the respondent State can prove that, without the measure actually adopted, the legitimate aim would not be achieved. Rather the core issue is whether, in adopting the measure under review, the competent authority acted within the margin of appreciation afforded to it $[\ldots]$.

To suggest that in a given situation only one option, namely the least restrictive one, is valid, without accepting that other, more restrictive options might be equally compatible with the Convention, amounts to disregarding the national authorities' margin of appreciation.

This is not to say that the availability of less restrictive measures is irrelevant for the assessment of whether the measure actually taken can be considered proportionate, and therefore 'necessary' [...]. The choice of the least restrictive measure is thus not a conditio sine qua non for the compatibility with the Convention of the measure adopted, but the failure to adopt a less restrictive measure is an element, among others, that may lead to the conclusion that the measure actually adopted was not 'necessary'. »

La démonstration ainsi livrée s'appuie sur un ensemble de précédents notables $^{70}$ où la Cour conteste que la condition de nécessité forme une exigence autonome dont l'absence de satisfaction conduirait ipso jure à la violation du principe de proportionnalité. Ainsi, dans l'arrêt National Union of Rail, Maritime and Transport Workers c. Royaume-Uni ${ }^{71}$, la Cour, s'expri-

69. Opinion concordante jointe à l'arrêt Gülbahar Özer and Ysuf Özer c. Turquie du 29 mai 2018. V. aussi l'opinion concordante de ce même juge jointe à CEDH, 18 avr. 2013, Saint-Paul Luxembourg SA c. Luxembourg.

70. CEDH, 21 févr. 1986, James et autres c. Royaume-Uni, § 86; 22 avr. 2013, Animal Defenders International c. Royaume-Uni, § 110; 6 nov. 2017, Garib c. Pays-Bas, § 157.

71. CEDH, 8 avr. 2014, National Union of Rail, Maritime and Transport Workers c. Royaume-Uni, $\S 103$. 
mant à propos de la compatibilité avec l'article 11 de l'interdiction totale des grèves de solidarité, énonce en guise de conclusion que:

«si l'histoire législative du Royaume-Uni montre qu'il pouvait exister des solutions autres qu'une interdiction totale, cela n'est pas déterminant pour trancher la question. En effet, la question n'est pas de savoir s'il aurait fallu adopter des règles moins restrictives ni même de savoir si l'État peut prouver que, sans pareille interdiction, le but légitime visé n'aurait pas pu être atteint. Il s'agit bien plutôt de déterminer si, lorsqu'il a adopté la mesure générale litigieuse, le législateur a agi dans le cadre de sa marge d'appréciation [...] - et si, globalement, il est parvenu à un juste équilibre.»

On le voit: neuf années après Glor, les hésitations demeurent possibles jusqu'au cœur des principes eux-mêmes. Le temps du colin-maillard complet est certes révolu, mais les tâtonnements subsistent.

\subsection{LES EXIGENCES PROCÉDURALES : GÉOMÉTRIES VARIABLES INEXPLIQUÉES}

Nous avons vu ci-dessus que, sur le plan des principes, la Cour européenne des droits de l'homme est disposée à censurer une mesure législative au motif que le débat qui a présidé à son adoption a manqué de profondeur, et n'a pas permis un échange d'arguments mûr et réfléchi. Cependant, et sur ce terrain également, s'observent de bien singulières éclipses. L'arrêt Belcacemi et Oussar c. Belgique ${ }^{72}$ en est un exemple significatif.

La Cour devait en l'espèce se prononcer sur la compatibilité avec l'article 9 de la Convention, lu isolément ou en combinaison avec l'article 14 du même instrument, de la loi belge du $1^{\text {er }}$ juin 2011 «interdisant le port de tout vêtement cachant totalement ou de manière principale le visage». L'arrêt, daté du 11 juillet 2017, résout cette question de manière affirmative. À l'appui de cette conclusion, il fait valoir, entre autres considérations, que «le processus décisionnel ayant débouché sur l'interdiction en cause a duré plusieurs années et a été marqué par un large débat au sein de la Chambre des représentants ainsi que par un examen circonstancié et complet de l'ensemble des intérêts en jeu par la Cour constitutionnelle ${ }^{73} »$. L'affirmation, que l'on retrouve également - à titre d'obiter dictum cette fois-ci - dans l'arrêt Dakir c. Belgique ${ }^{74}$, laisse relativement songeur. Ainsi que le fit observer le

72. CEDH, 11 juill. 2017, Belcacemi et Oussar c. Belgique.

73. Ibid., \& 54 .

74. CEDH, 11 juill. 2017, Dakir c. Belgique. 
Centre des droits de l'homme de l'Université de Gand, tiers-intervenant en l'affaire Dakir ${ }^{75}$, le processus d'adoption de la loi précitée du $1^{\text {er }}$ juin 2011, tout «long» qu'il ait été, ne fut pas vraiment caractérisé par sa profondeur et le souci de ses protagonistes de documenter leur dossier, en fait et en droit ${ }^{76} \ldots$ Les demandes d'audition des représentants du milieu associatif, très critique à l'égard de la loi en devenir, furent systématiquement repoussées ${ }^{77}$. Les députés ne jugèrent pas non plus utile d'entendre les femmes portant un voile intégral. Last but not least, la Chambre des représentants refusa à plusieurs reprises de consulter, pour avis, la Section de législation du Conseil d'État. Un député, le plus sérieusement du monde, justifia ce refus en affirmant qu'il ne "souhait[ait] en aucun cas s'engager dans un débat qui consiste à vérifier si l'interdiction du port de la burqa ou d'autres vêtements du genre viole le droit international ou le droit européen, auquel cas ceux qui sont en faveur de la burqa pourraient gagner ce combat ${ }^{78} »$. On peine vraiment à qualifier pareille discussion parlementaire de "profonde», au sens de l'arrêt Hirst ( $\left.n^{\circ} 2\right) \ldots$ La conclusion en sens contraire de la Cour, qui pourtant s'était vue mettre sous les yeux tous les éléments d'appréciation utiles de la part du Centre des droits de l'homme de l'Université de Gand, est donc pour le moins surprenante. On pourrait être tenté de remettre cette approche peu critique du processus législatif belge sur le compte de l'existence du précédent français. Aurait-il été parfaitement cohérent de censurer dans le chef de la Belgique (Belcacemi et Oussar; Dakir) un "choix législatif » dont la substance avait été admise trois ans auparavant dans le chef de la France (S.A.S), pour le seul motif que la délibération parlementaire menée à Bruxelles avait été notablement plus bâclée et moins informée que celle conduite à Paris? Aussi contre-intuitif qu'il puisse paraître, la réponse à cette question n'est pas nécessairement négative. Les députés belges auraient-ils en effet nécessairement voté cette loi si, moyennant une meilleure information du débat - par exemple via des auditions ou la consultation de la Section de législation du Conseil d'État -, ils avaient eu conscience que l'interdiction de la dissimulation du visage dans l'espace public ne peut en tous cas pas s'autoriser d'impératifs de préservation de l'ordre public matériel, ni du

75. Le texte intégral de la note du tiers-intervenant peut être consulté en ligne: http://www. hrc.ugent.be/wp-content/uploads/2015/11/Dakir_hrc.pdf [consulté le 18 déc. 2018].

76. Pour une analyse en profondeur des travaux parlementaires, V. X. Delgrange, "Quand la Burqa passe à l'Ouest, la Belgique perd-t-elle le Nord?», in D. Koussens (dir.), Quand la burqa passe à l'Ouest. Enjeux éthiques, politiques et juridiques, Rennes, PUR, 2013, p. 200 et $\mathrm{s}$.

77. Ibid., p. 202

78. Cité ibid., p. 201. 
principe de l'égalité entre les hommes et les femmes? Peut-être. Ou peut-être pas. La Cour européenne des droits de l'homme n'avait en tous cas pas à présumer qu'un meilleur débat et une autre pesée des intérêts réalisés au Parlement aurait de toute façon conduit à un résultat identique à celui in fine atteint: une telle «substitution de motifs» est en effet aux antipodes de l'idée même de marge nationale d'appréciation.

Il fut un temps où les travaux doctrinaux dédiés au principe de proportionnalité se soldaient par une franche perplexité. Ce n'était pas dans les détails et sous-questions que fleurissaient les doutes et les controverses, mais bien sûr les observations de base. Cette époque est - croyons-nous - révolue: un réel effort pédagogique a été produit par la Cour pour dire plus explicitement ce qu'elle fait lorsqu'il est question de proportionnalité, et déplier ainsi de manière plus transparente les multiples exigences, substantielles et procédurales que coiffe ce principe, ainsi que les liens qui les unissent. Loin s'en faut, cependant, que la proportionnalité ait révélé ainsi tous ses mystères: l'effort reste à poursuivre. 